\title{
PATHOLOGY AND BIOLOGY
}

\author{
Amanda O. Fisher-Hubbard, ${ }^{1}$ M.D.; Kilak Kesha, ${ }^{1,2}$ M.D.; Francisco Diaz, ${ }^{1,2}$ M.D.; Chantel Njiwaji, ${ }^{1,2}$ M.D.; \\ PaoLin Chi, ${ }^{3}$ M.D.; and Carl J. Schmidt, ${ }^{1,2}$ M.D.
}

\section{Commode Cardia_-Death by Valsalva Maneuver: A Case Series*}

\begin{abstract}
The Valsalva maneuver is used in clinical medicine for the diagnosis and/or treatment of various cardiovascular conditions. It can also be used in activities of daily living, such as defecation. Due to the cardiovascular effects produced during the Valsalva maneuver, it may be contraindicated in certain medical conditions and could be a trigger of sudden cardiac death. The incidence and prevalence of death following Valsalva maneuver in the presence of underlying cardiovascular disease, or "commode cardia," has not been examined. In 2012, the Wayne County Medical Examiner's Office (Detroit, MI) investigated 21 deaths that occurred on the toilet, fourteen of which were due to cardiovascular disease. In another 31 deaths in the bathroom due to cardiovascular disease, the possibility that the decedent defecated immediately prior to death could not be excluded. Hence, the incidence of commode cardia in this population ranges from 2.3 to $7.4 \%$ of all cardiovascularrelated deaths.
\end{abstract}

KEYWORDS: forensic science, forensic pathology, Valsalva maneuver, vagal maneuver, sudden cardiac death, cardiovascular disease, commode cardia

The Valsalva maneuver is a sustained, forced expiration against the resistance of a closed nose or glottis. It was first described in 1704 by Antonio Maria Valsalva (1666-1723), a professor of anatomy at the University of Bologna, surgeon to the Hospital for Incurables, and President of the Academy of Sciences. Valsalva was particularly interested in the human ear. In his only published paper, De aure humana tractatus, he described the detailed anatomy of the ear, as well as a method for clearing the eustachian tube. This method is what we now refer to as the Valsalva maneuver (1-4). Approximately 150 years later, the cardiovascular responses that occur during the Valsalva maneuver were first described by Ernst Heinrich Weber $(1,5)$. Since that time, the physiologic effects of the maneuver have been extensively studied. It is considered a vagal maneuver, as it stimulates the vagus nerve and activates the parasympathetic nervous system. It is used in modern cardiovascular medicine to differentiate various heart murmurs, diagnose congestive heart failure and autonomic disorders, and treat certain arrhythmias $(2,3)$. In addition, it is often used during normal daily activities, such as defecation, sneezing, coughing, or heavy lifting (1).

The Valsalva maneuver is divided into four phases (Fig. 1). In phase I (onset of strain), there is an increase in both intrathoracic

${ }^{1}$ Department of Pathology, University of Michigan, 1301 Catherine Street, Ann Arbor, MI 48109.

${ }^{2}$ Wayne County Medical Examiner's Office, 1300 E. Warren Avenue, Detroit, MI 48207.

${ }^{3}$ Integrated Healthcare Association, 3100 E. Eisenhower Parkway, Suite 100, Ann Arbor, MI 48108.

* Presented at the 66th Annual Meeting of the American Academy of Forensic Sciences, February 17-20, 2014, in Seattle, WA.

Received 7 Aug. 2015; and in revised form 24 Nov. 2015; accepted 6 Feb. 2016 pressure and systolic blood pressure due to compression of the aorta. In addition, there is a small but significant decrease in coronary artery blood flow. In phase II (continued strain), there is a decrease in venous return and thus a decrease in both cardiac output and systolic blood pressure. In addition, there is a reflex tachycardia. In phase III (release of strain), there is a decrease in intrathoracic pressure and thus expansion of the aorta and the pulmonary vasculature. This leads to further decreases in cardiac stroke volume and systolic blood pressure. In phase IV (recovery), there is an "overshoot" in systolic blood pressure from both increased cardiac output and sympathetically mediated vasoconstriction. As a result, there is a reflex bradycardia $(2,3,6)$.

Due to these complex, physiologic changes induced by the Valsalva maneuver, it may be contraindicated in a variety of cardiovascular conditions, including hypertrophic obstructive cardiomyopathy, significant aortic valvular disease, recent myocardial infarction, and aortic stenosis. It is possible that individuals with these cardiovascular conditions who perform the Valsalva maneuver during defecation could suffer a number of adverse events. These include a syncopal episode through decreased cerebral blood flow and cerebral perfusion, sinus pause, atrioventricular block, arrhythmia, or myocardial infarct from decreased flow through the coronary arteries $(1,5,6)$. In individuals with arteriosclerotic heart disease, these processes could severely affect the already tenuous cardiac blood supply. Thus, the Valsalva maneuver can precipitate a potentially fatal chain of events.

In the late 1940s, McGuire et al. addressed the "notorious frequency of sudden and unexpected deaths of patients while using bed pans in hospitals (7)." They reviewed 6700 hospital autopsy protocols from postmortem examinations performed at Cincinnati General Hospital over a 10-year period. They identified eleven 


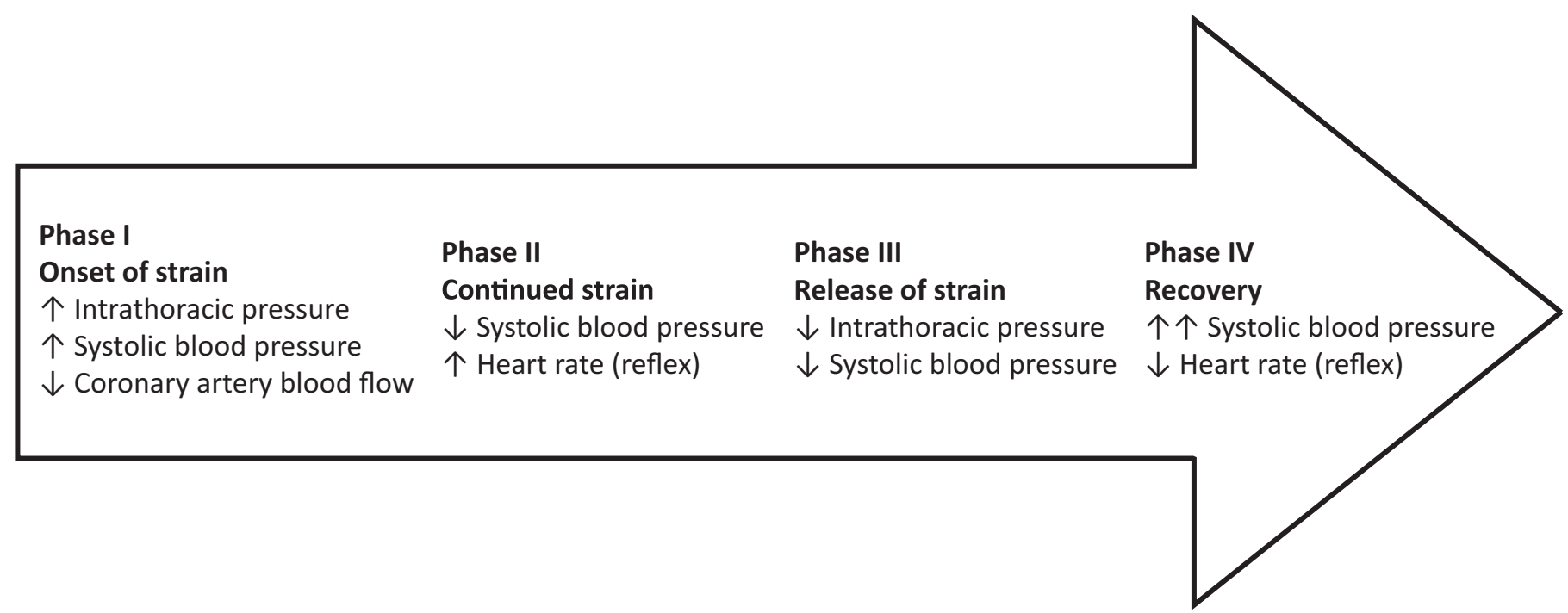

FIG 1-Physiologic changes during the Valsalva maneuver.

deaths that occurred immediately following the use of a hospital bed pan. Of these decedents, ten had underlying organic heart disease (7). These investigators stated that these patients died "suddenly and unexpectedly" while using the bedpan in a hospital (7). However, most medical examiners would argue that in many ill, hospitalized patients, sudden deaths are not necessarily unexpected. This study, therefore, does have limitations. Although the phenomenon of sudden cardiac arrest and death following the Valsalva maneuver has been documented, little is known about its incidence and prevalence (5,7-9). In addition, there is no universally accepted term from this occurrence.

We hypothesize that the Valsalva maneuver, particularly in the setting of defecation, is a trigger of sudden cardiac death in the presence of underlying cardiovascular disease. The authors propose the term "commode cardia" to describe this phenomenon.

\section{Methods}

We performed a retrospective review of deaths investigated by the Wayne County Medical Examiner's Office (Detroit, MI) in 2012 to identify deaths that occurred in the bathroom. We queried the case management database maintained by the office using the keywords "bathroom," "washroom," "restroom," "water closet," "toilet," "commode," "lavatory," "latrine," and "privy." We then reviewed the scene investigation and autopsy reports in those cases to identify the deaths that occurred on the toilet. In these cases, the pathologists used medical death investigators' reports and postmortem examination findings to form an opinion regarding cause of death. The extent of the examination was at the discretion of the medical examiner. We also identified the cases in which the death occurred in the bathroom and in which the possibility that the decedent died on the toilet could not be excluded. To capture the latter set of cases, the following inclusion criteria were used: the decedent was discovered beside the toilet with his/her undergarment and/or pants (if not wearing an undergarment) pulled down and/or there was excrement in the toilet. We excluded cases in which the decedent was found nude next to the toilet without evidence of excrement. We noted the cause and manner of death in each case. In each death due to cardiovascular disease (arteriosclerotic cardiovascular disease,

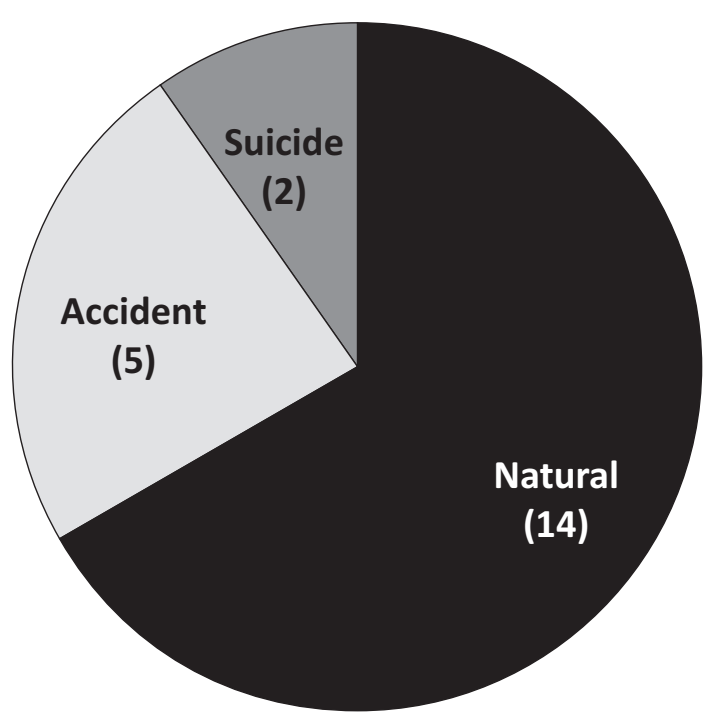

FIG 2-Deaths that occurred on the toilet, by manner $(n=21)$.

hypertensive cardiovascular disease, or hypertensive arteriosclerotic cardiovascular disease), we recorded the age, race, sex, height, weight, body mass index, and clinical history of the decedent. Regarding clinical history, we paid particular attention to cardiovascular disease and its risk factors, such as obesity, diabetes mellitus, hyperlipidemia, and hypertension (10).

\section{Results}

In 2012, the Wayne County Medical Examiner's Office in Detroit, MI, investigated a total of 2766 deaths. Of those cases, 127 deaths occurred in the bathroom. Twenty-one individuals died on the toilet (Fig. 2): fourteen of cardiovascular disease (natural), four of drug intoxication (accident), one of incised wounds to the wrist (suicide), one of a gunshot wound to the head (suicide), and one of smoke and soot inhalation (accident).

The demographics of the fourteen decedents who died naturally are summarized in Table 1. Examples of scene investigation photographs are shown in Figs 3 and 4. After appropriate 
TABLE 1-Demographics of commode cardia deaths.

\begin{tabular}{|c|c|c|}
\hline & Discovered on toilet & $\begin{array}{l}\text { Discovered on } \\
\text { or near toilet* }\end{array}$ \\
\hline Number of cases & 14 & 45 \\
\hline Age $\left(\right.$ years) ${ }^{\dagger}$ & $65.6(29-94)$ & 63 (29-94) \\
\hline Male:Female & $11: 3$ & $7: 2$ \\
\hline Black:White & $5: 2$ & $29: 16$ \\
\hline Height (inches) ${ }^{\dagger}$ & $68.6(60-72)$ & $68.7(57-75)$ \\
\hline Weight (pounds) ${ }^{\dagger}$ & $162.1(100-267)$ & $167.7(98-368$ \\
\hline Body mass index (BMI; $\left.\mathrm{kg} / \mathrm{m}^{2}\right)^{\dagger}$ & $24.2(13.6-39.4)$ & $24.9(13.6-46)$ \\
\hline Cardiovascular disease $(\%)^{\ddagger}$ & 64.3 & 68.9 \\
\hline
\end{tabular}

*Includes those discovered on the toilet (14 decedents) and those discovered near the toilet (31 decedents).

${ }^{\dagger}$ Average (range).

"Includes presence of clinical history, modifiable risk factors, and/or risk equivalents as per the American Heart Association and American College of Cardiology (e.g., coronary artery disease, myocardial infarction, hypertension, diabetes, obesity, hyperlipidemia, congestive heart failure, cardiomyopathy) (9).

medical death investigations, the medical examiners determined that all fourteen of these deaths were due to cardiovascular disease. There was no evidence of an acute myocardial infarct or acute coronary artery thrombus noted in any of the decedents. Complete histopathological examinations were performed on 6 of the $14(43 \%)$ decedents found on the toilet. The average age of these decedents was 65.6 years, although the range was from 29 to 94 years. The average body mass index of these decedents was within the normal range $\left(18.5-24.9 \mathrm{~kg} / \mathrm{m}^{2}\right)(11)$. In almost two-thirds of the deaths, the decedent had a clinical history of cardiovascular disease and/or at least one risk factor for cardiovascular disease, including obesity.

In another 31 cases of death due to cardiovascular disease, the possibility that the decedent used the toilet just prior to having a fatal cardiac event could not be excluded. The demographics of these decedents plus those who died on the toilet are detailed in Table 1. As in those found on the toilet, there was no evidence of an acute myocardial infarct or acute coronary artery thrombus noted in any of the decedents. Complete histopathological examinations were performed on 9 of the $31(29 \%)$ decedents discovered beside or near the toilet. The average age of these decedents was 63 years, although the range was unaffected by the addition of these cases. The average body mass index remained within the normal range. In just over two-thirds of the deaths, the decedent had a clinical history of cardiovascular disease and/or a cardiovascular disease risk factor.

There were eighteen additional natural deaths that occurred in the bathroom during this time period in which the possibility that the decedent used the toilet just prior to death was excluded. Of these cases, there were five deaths due to cardiovascular disease.

During that same year, the office investigated a total of 608 cases of arteriosclerotic cardiovascular disease, hypertensive cardiovascular disease, and hypertensive arteriosclerotic cardiovascular disease. Based on these data, the incidence of Valsalva maneuver during defecation immediately preceding a terminal cardiac event, or commode cardia, is between $2.3 \%$ and $7.4 \%$ in those who died of arteriosclerotic cardiovascular disease, hypertensive cardiovascular disease, or hypertensive arteriosclerotic cardiovascular disease.

\section{Discussion}

Cardiovascular disease is the leading cause of death for both men and women in the United States. It is estimated that over

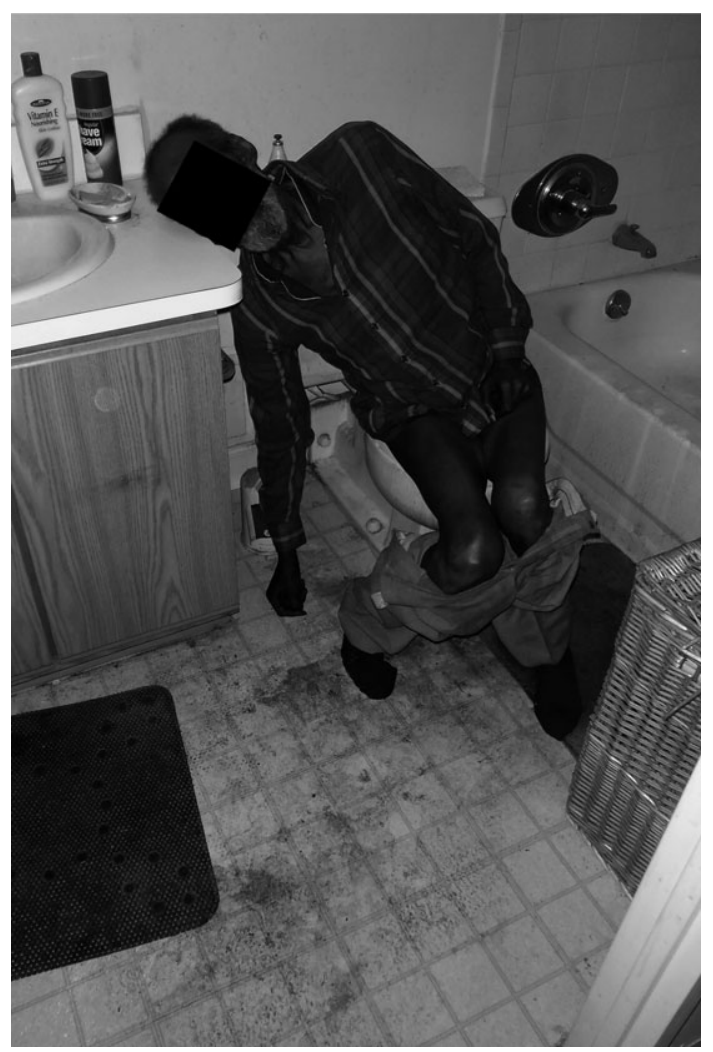

FIG 3-Seventy-six-year-old black male with a history of hypertension. Cause of death: arteriosclerotic cardiovascular disease. Manner of death: natural.

80 million, or 1 in 3 , Americans have at least one form of cardiovascular disease. The overall rate of death attributed to cardiovascular disease in 2010 was 235.5 per 100,000. Based on this rate, more than 2000 Americans die of cardiovascular disease each day. Coronary heart disease alone caused approximately one of every six deaths in the United States in 2010 (12). These individuals are at risk for sudden cardiac death (13).

Sudden cardiac death is defined as an unexpected natural death from a cardiac etiology within a short period of time (generally an hour or less) from the onset of symptoms in a person without any prior condition that would appear fatal (13). These deaths generally occur in people with structural heart diseases. It is the most common and often first manifestation of coronary heart disease. Because the majority of sudden cardiac deaths occur in individuals with coronary disease, the risk factors for both are similar and include increased age, male sex, glucose intolerance or diabetes, obesity, smoking, hypertension, and hyperlipidemia (13).

In addition to these modifiable and nonmodifiable "chronic" risk factors, there are transient, "acute" risk factors for sudden cardiac death and/or acute cardiovascular disease. These acute risk factors are temporary physiological changes (increases in arterial pressure or heart rate, increased coagulability, or vasoconstriction) that follow a trigger or event and may result in disease onset or death $(14,15)$. Triggers include emotional stress (anxiety, anger, grief), natural disasters (earthquakes), physical activity (including sexual activity), air pollution, respiratory infections, heavy meals, lack of sleep, and drugs (cocaine, marijuana) (14-18). Furthermore, strategies have been developed to mitigate and/or avoid these activities, an approach known as 


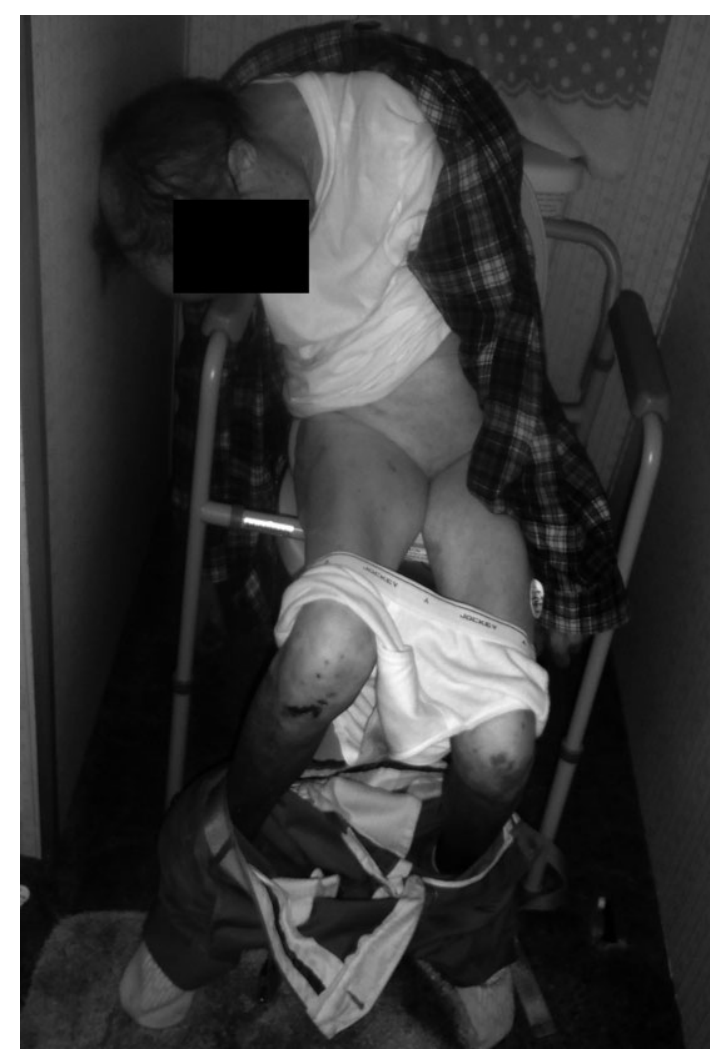

FIG 4-Sixty-seven-year-old white male with a history of hypertension. Cause of death: arteriosclerotic cardiovascular disease. Manner of death: natural.

"triggered acute risk prevention" (TRAP) (15). These include trigger-specific preventive therapies (e.g., stress-reduction techniques, vaccinations), assessment of the absolute risk of an individual patient, avoidance or modification, and use of medications (15).

The Valsalva maneuver during defecation is a trigger of sudden cardiac arrest and death that has been documented but not extensively investigated (5,7-9). The epidemiology of death following defecation, what the authors refer to as commode cardia, is unclear. An evaluation of the phases of the Valsalva maneuver reveals the complex physiologic changes produced during this event (Fig. 1). Many of the same cardiovascular alterations that occur with the aforementioned triggers also occur during the Valsalva maneuver. Bearing in mind the prevalence of cardiovascular disease in the United States and the frequency at which one might perform the Valsalva maneuver, it is conceivable that commode cardia is a legitimate public health concern.

Our study suggests that all natural deaths that occurred on the toilet were due to commode cardia in this population. Additionally, we identified another set of deaths that occurred in the bathroom in which possibility that the decedent died on the toilet could not be excluded. Overall, the incidence of commode cardia in our population was up to $7 \%$ of all deaths attributed to cardiovascular disease in 2012. When we look at these groups, we see that these individuals are predominately older males with normal body mass indices. In approximately two-thirds of these deaths, the decedents had documented clinical histories, modifiable risk factors, and/or risk equivalents for cardiovascular disease. Some risk factors, such as smoking history, dietary habits, and activity level, were unknown. Thus, it is likely that we underestimated the percentage of decedents with underlying risk factors.

We propose that commode cardia is a phenomenon that should be discussed with cardiac patients. Specific attention should be paid to those with coronary heart disease and to those who have suffered a myocardial infarction. These patients should be counseled by clinicians in a nonthreatening, nonalarmist manner, just as they are advised regarding physical activity, sexual activity, stress management, and lifestyle modifications. Clinicians might consider adding stool softeners and/or laxatives to the treatment regimens of their patients. Opioid pain medications and iron supplementation should also be used judiciously in this population, as these may lead to constipation and thus unnecessary straining. Additionally, modifications to the traditional defecation posture, such as squatting instead of sitting, have been suggested by other investigators (8). A squatting posture has been associated with reduced straining efforts, as it more effectively straightens the recto-anal angle than sitting and thus leads to easier evacuation of stool (8).

This is the first study that has attempted to address the incidence of commode cardia. There are, however, limitations to our study. Most of these limitations are due to the fact that these deaths are typically unwitnessed. As we excluded anyone who was found nude next to the toilet with no evidence of excrement, we might have excluded additional cases of commode cardia in which the Valsalva maneuver occurred without evacuation of stool. Additionally, individuals who are suffering from a cardiac event may feel ill and subsequently use the toilet in an attempt to relieve the symptoms. These latter cases are not true commode cardia cases, as the cardiac event would have preceded the Valsalva maneuver. It is worth reiterating, however, that the Valsalva maneuver causes measurable cardiovascular alterations, including variations in blood pressure and decreased coronary blood flow. Therefore, if an individual felt ill and tried to relieve his or her symptoms by attempting to defecate, it is possible that the Valsalva maneuver might have contributed to a fatal cardiac arrhythmia. We are faced with these limitations because the sequence of events in these unwitnessed deaths cannot be determined at the time of postmortem examination. To prove causation would require controlled experiments with monitoring of vital signs and electrocardiography.

\section{Conclusions}

We postulate that the Valsalva maneuver during defecation is a trigger of sudden cardiac death in the presence of underlying cardiovascular disease. The authors propose the term "commode cardia" to describe this phenomenon. Commode cardia should be considered by medical examiners and coroners in the investigation of sudden cardiac deaths that occur in the bathroom. Clinicians should appropriately address the potential implications of the Valsalva maneuver with patients who may be at risk. Methods to avoid or modify this particular vagal maneuver should be discussed in the clinical setting.

\section{Acknowledgment}

The authors would like to acknowledge Gerry Polverento, information technology and photography department manager and health surveillance officer at the Wayne County Medical Examiner's Office, who provided assistance in queries of the case management database. 


\section{References}

1. Jellinek EH. The Valsalva manoeuvre and Antonio Valsalva (16661723). J R Soc Med 2006;99(9):448-51.

2. Arrigo M, Huber LC. Eponyms in cardiopulmonary reflexes. Am J Cardiol 2013;112(3):449-53.

3. Yale SH. Antonio Maria Valsalva (1666-1723). Clin Med Res 2005;3 (1):35-8.

4. Valsalva AM. De aure humana tractatus. Utrecht, the Netherlands: van de Water, 1707.

5. Looga R. The Valsalva manoeuvre - cardiovascular effects and performance technique: a critical review. Respir Physiol Neurobiol 2005;147 (1):39-49.

6. Benchimol A, Wang TF, Desser KB, Gartlan JL Jr. The Valsalva maneuver and coronary arterial blood flow velocity. Studies in man. Ann Intern Med 1972;77(3):357-60.

7. McGuire J, Green RS, Courter S, Hauenstein V, Braunstein JR, Plessinger V, et al. Bed pan deaths. Trans Am Clin Climatol Assoc 1948;60:78-86.

8. Sikirov BA. Cardio-vascular events at defecation: are they unavoidable? Med Hypotheses 1990;32(3):231-3.

9. Schartum S. Ventricular arrest caused by the Valsalva maneuver in a patient with Adams-Stokes attacks accompanying defecation. Acta Med Scand 1968;184(1-2):65-8.

10. Eckel RH, Jakicic JM, Ard JD, de Jesus JM, Houston Miller N, Hubbard VS, et al. 2013 AHA/ACC guideline on lifestyle management to reduce cardiovascular risk: a report of the American College of Cardiology/ American Heart Association Task Force on Practice Guidelines. Circulation 2014;129(25 Suppl 2):S76-99.
11. Pi-Sunyer FX, Becker DM, Bouchard C, Carleton RA, Colditz GA, Dietz $\mathrm{WH}$, et al. Clinical guidelines on the identification, evaluation, and treatment of overweight and obesity in adults - the evidence report. National Institutes of Health. Obes Res 1998;6(Suppl 2):51S-209S.

12. Go AS, Mozaffarian D, Roger VL, Benjamin EJ, Berry JD, Blaha MJ, et al. Heart disease and stroke statistics - 2014 update: a report from the American Heart Association. Circulation 2014;129(3):e28-292.

13. Zipes DP, Wellens HJ. Sudden cardiac death. Circulation 1998;98 (21):2334-51.

14. Muller JE, Tofler GH, Stone PH. Circadian variation and triggers of onset of acute cardiovascular disease. Circulation 1989;79 (4):733-43.

15. Tofler GH, Muller JE. Triggering of acute cardiovascular disease and potential preventive strategies. Circulation 2006;114(17):1863-72.

16. Muller JE. Sexual activity as a trigger for cardiovascular events: what is the risk? Am J Cardiol 1999;84(5B):2N-5N.

17. Culić V. Acute risk factors for myocardial infarction. Int J Cardiol 2007;117(2):260-9.

18. Schwartz BG, French WJ, Mayeda GS, Burstein S, Economides C, Bhandari AK, et al. Emotional stressors trigger cardiovascular events. Int J Clin Pract 2012;66(7):631-9.

Additional information and reprint requests:

Amanda O. Fisher-Hubbard, M.D.

Western Michigan University

Homer Stryker M.D. School of Medicine

1000 Oakland Drive

Kalamazoo, MI 49008

E-mail: amanda.fisher-hubbard@med.wmich.edu 